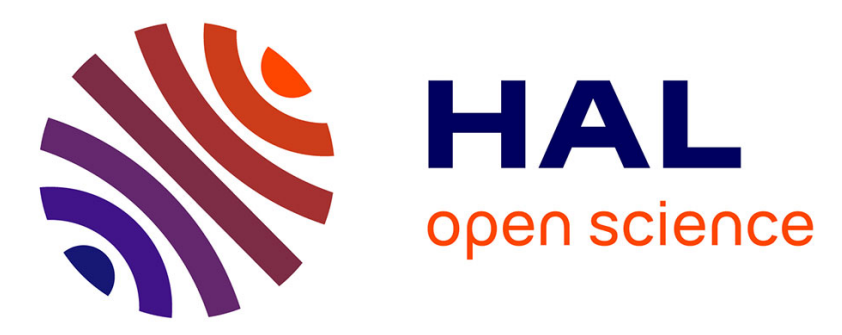

\title{
Numerical modelings of ultrashort pulse propagation and conical emission in multimode optical fibers
}

\author{
Karol Tarnowski, Sylwia Majchrowska, P. Béjot, Bertrand Kibler
}

\section{To cite this version:}

Karol Tarnowski, Sylwia Majchrowska, P. Béjot, Bertrand Kibler. Numerical modelings of ultrashort pulse propagation and conical emission in multimode optical fibers. Journal of the Optical Society of America B, 2021, 38, pp.732-742. 10.1364/JOSAB.413050 . hal-03066147

\section{HAL Id: hal-03066147 \\ https://hal.science/hal-03066147}

Submitted on 15 Dec 2020

HAL is a multi-disciplinary open access archive for the deposit and dissemination of scientific research documents, whether they are published or not. The documents may come from teaching and research institutions in France or abroad, or from public or private research centers.
L'archive ouverte pluridisciplinaire HAL, est destinée au dépôt et à la diffusion de documents scientifiques de niveau recherche, publiés ou non, émanant des établissements d'enseignement et de recherche français ou étrangers, des laboratoires publics ou privés. 


\title{
Numerical modelings of ultrashort pulse propagation and conical emission in multimode optical fibers
}

\author{
Karol Tarnowski ${ }^{1,}$, Sylwia Majchrowska ${ }^{1}$, Pierre BÉjot ${ }^{2}$, and Bertrand KibleR ${ }^{2}$ \\ ${ }^{1}$ Department of Optics and Photonics, Wroclaw Univeristy of Science and Technology, Wybrzeze Wyspianskiego 27, Wroclaw 50-370, Poland \\ ${ }^{2}$ Laboratoire Interdisciplinaire Carnot de Bourgogne (ICB), UMR6303 CNRS-Université Bourgogne Franche-Comte, 21078 Dijon, France \\ *Corresponding author: karol.tarnowski@pwr.edu.pl
}

Compiled December 15, 2020

\begin{abstract}
We make use of two well-known numerical approaches of nonlinear pulse propagation, namely the unidirectional pulse propagation equation and the multimode generalized nonlinear Schrödinger equation, to provide a detailed comparison of ultrashort pulse propagation and possible conical emission in the context of multimode optical fibers. We confirm the strong impact of the frequency dispersion of the nonlinear response on pulse splitting and supercontinuum dynamics in the femtosecond regime for pumping powers around the critical self-focusing threshold. Our results also confirm that the modal distribution of optical fibers provides a discretization of conical emission of the corresponding bulk medium (i.e., here fused silica). This study also provides some criteria for the use of numerical models and it paves the way for future nonlinear experiments in commercially-available optical fibers. (๑) 2020 Optical Society of America
\end{abstract}

http://dx.doi.org/10.1364/ao.XX.XXXXXX

\section{INTRODUCTION}

Nonlinear light propagation in multimode fibers (MMFs) has recently attracted a surge of interest $[1,2]$ through the investigation of complex linear and nonlinear modal interactions provided by the added spatial degrees of freedom when compared to single-mode waveguides. In that respect, recent observations include ultrabroadband supercontinuum generation, Kerr and Raman beam cleaning, as well as intermodal four-wave mixing processes to name a few [2-4]. The motivation of mentioned works is usually two-fold: (i) a deeper understanding of nonlinear optics, and (ii) to address the great expectations concerning the application of multimode fibers for high-capacity communication networks [5, 6] and for high-energy fiber lasers [3, 7].

However, most of the time, the derived numerical models only support qualitatively experimental results, in particular, when the number of modes involved becomes significant (i.e., higher than 10) and ultrabroadband frequency conversion processes (i.e., more than one octave) also occur $[8,9]$. Basically, two different approaches can be mentioned, namely the multimode generalized nonlinear Schrödinger equation (MM-GNLSE) $[10,11]$ and the Gross-Pitaevskii equation (GPE) [12-14], even if some related alternatives were also investigated $[15,16]$. The first one considers a modal decomposition of the electrical field and then intermodal nonlinear couplings, whereas as the second one is based on a direct representation of the electric field in the space-time domain and the refractive index distribution acts as a potential term. As already noted in a few papers [17, 18], for both approaches, strong approximations are present in the propagation equation used that can lead to significant divergences from the exact case. For instance, such limiting issues have been already solved during the last decade in the case of simpler modelings related to light propagation in single-mode highly nonlinear waveguides and fibers. In that case, the standard nonlinear Schrödinger equation (NLSE) is usually extended with higher-order linear and nonlinear terms to give rise to generalized versions of the NLSE $[19,20]$. In other words, their derivation from the Maxwell equations contain less approximations, and such models usually follow a common structure that originate from the unidirectionnal pulse propagation equation (UPPE) $[8,21,22]$. The same approach can be applied to multimode systems through the UPPE generalized to structure media $[17,18]$. One can derive the MM-GNLSE and GPE from generalized UPPE. It is worth mentioning that numerical models based on UPPE have been widely used for studying nonlinear optics in bulk transparent media and gases [23-25]. Among many nonlinear spatiotemporal phenomena, conical emission, femtosecond filamentation, harmonic generation and light bullets remain some of the striking phenomena that can be described [26].

In this paper, we compare two modeling approaches in a highly nonlinear regime of pulse propagation in multimode fibers, which corresponds to femtosecond pulse pumping with peak power close the critical self-focusing power of silica glass [27]. Indeed, there is an urgent need of clarifying the potential and areas of applicability of numerical models to facilitate the accurate design of future nonlinear and multimode fibers [28-31]. As a consequence, the numerical models under study 
are here the MM-GNLSE, well-known in the nonlinear fiber community [10,11], and the multimode unidirectional pulse propagation equation (MM-UPPE) [18]. This easily-accessible pumping regime, from the experimental point of view, appears as a good starting point to reveal some limitations of approximated numerical models and novel alluring phenomena in optical fibers. A detailed comparison of ultrashort pulse propagation is provided in the case of commercially-available multimode step-index fibers. It clearly reveals the strong impact of the dispersion of the nonlinearity on pulse splitting and supercontinuum dynamics in the femtosecond regime. Moreover, we show that the resulting spatiotemporal dynamics are associated with conical wave formation.

This article is organized as follows, first we recall the background of numerical tools used to describe optical pulse propagation in the scalar approximation, and we briefly describe the fiber properties under study (see Section 2). Next, we simultaneously present the results of both numerical models of high-power femtosecond pulse propagation in a short segment of standard multimode step-index fiber. More specifically, we compare the available information provided by each approach and analyze the discrepancies on pulse dynamics in space-time and frequency domains as a function of modeling assumptions (see Section 3). Finally, we investigate in more detail the formation of $\mathrm{X}$-waves as a function of the fiber core diameter (see Section 4).

\section{NUMERICAL MODELS AND FIBER'S PARAMETERS}

\section{A. Multimode unidirectional pulse propagation equation}

We first recall the linear wave equation, which writes in the frequency domain:

$$
\left[\triangle_{\perp}+\frac{n^{2}(\vec{r}, \omega) \omega^{2}}{c^{2}}+\partial_{z}^{2}\right] \overrightarrow{E_{\omega}}-\vec{\nabla}\left(\vec{\nabla} \cdot \overrightarrow{E_{\omega}}\right)=\overrightarrow{0}
$$

In the following, we consider only optical waveguides whose refractive indices are invariant along the $z$ direction. Optical modes are electromagnetic fields that are solutions of Eq.1 that write:

$$
\vec{E}\left(\overrightarrow{r_{\perp}}, \omega, z\right)=\vec{A}\left(\overrightarrow{r_{\perp}}, \omega\right) e^{i \beta(\omega) z},
$$

where $\overrightarrow{r_{\perp}}$ is a vector perpendicular to the propagation axis $z$. In the weak guidance approximation (neglecting the second term in the above equation), Eq.1 can be simplified in its scalar version:

$$
\left[\triangle_{\perp}+\frac{n^{2}\left(\overrightarrow{r_{\perp}}, \omega\right) \omega^{2}}{c^{2}}+\partial_{z}^{2}\right] E_{\omega}=0 .
$$

Accordingly, the modes are transverse, i.e., their polarizations are contained in the plane perpendicular to the propagation axis $z$. For clarity, we will limit our discussion here to linearly polarized modes. Injecting Eq.2 in Eq.3, one obtains

$$
\begin{aligned}
{\left[\triangle_{\perp}+\frac{n^{2}\left(\overrightarrow{r_{\perp}}, \omega\right) \omega^{2}}{c^{2}}\right] A\left(\overrightarrow{r_{\perp}}, \omega\right) } & =\beta^{2}(\omega) A\left(\overrightarrow{r_{\perp}}, \omega\right) \\
\square_{\perp} A\left(\overrightarrow{r_{\perp}}, \omega\right) & =\beta^{2}(\omega) A\left(\overrightarrow{r_{\perp}}, \omega\right) .
\end{aligned}
$$

Optical modes are then eigenvectors of the operator $\square_{\perp}$ with the eigenvalue $\beta^{2}$. Structured and/or finite media (i.e., waveguides) support only a discrete (but infinite) number of modes at a given frequency $\omega$. Among all modes, one can exhibit three distinct families: core-guided, clad-guided, and evanescent modes. Moreover, since the number of modes is discrete, they can be sorted and indexed by a number $m$. A mode is then unambiguously identified by the couple $(m, \omega)$ and writes in the spatiotemporal domain as:

$$
\varepsilon\left(\overrightarrow{r_{\perp}}, t, m, \omega\right)=\mathcal{F}\left(\overrightarrow{r_{\perp}}, m, \omega\right) e^{-i \omega t},
$$

where $\mathcal{F}$ is the transverse shape of the $\mathrm{m}^{\text {th }}$ mode at the frequency $\omega$. Moreover, they are orthogonal with respect to the following scalar product:

$$
\iiint \varepsilon\left(\overrightarrow{r_{\perp}}, t, m, \omega\right) \varepsilon\left(\vec{r}_{\perp}, t, m^{\prime}, \omega^{\prime}\right)^{*} r d r d \theta d t=\delta_{m, m^{\prime}} \delta\left(\omega-\omega^{\prime}\right) .
$$

The modes $\varepsilon\left(\vec{r}_{\perp}, t, m, \omega\right)$ form an orthogonal basis set for representing the electric field $E\left(\overrightarrow{r_{\perp}}, t\right)$ :

$$
E\left(\overrightarrow{r_{\perp}}, t\right)=\int \sum_{m} \bar{E}(m, \omega) \varepsilon\left(\overrightarrow{r_{\perp}}, t, m, \omega\right) d \omega,
$$

where $\bar{E}(m, \omega)$ is a function representing the weight (i.e., the coordinate) of the $m^{\text {th }}$ mode at the frequency $\omega$ of the field decomposition in the modal basis. Moreover, $\bar{E}(m, \omega)$ can be evaluated as:

$$
\begin{aligned}
\bar{E}(m, \omega) & =\iiint E\left(\overrightarrow{r_{\perp}}, t\right) \varepsilon\left(\overrightarrow{r_{\perp}}, t, m, \omega\right)^{*} r d r d \theta d t \\
& =\iint \widetilde{E}\left(\overrightarrow{r_{\perp}}, \omega\right) \mathcal{F}\left(\overrightarrow{r_{\perp}}, m, \omega\right) r d r d \theta,
\end{aligned}
$$

where $\widetilde{E}\left(\overrightarrow{r_{\perp}}, \omega\right)$ is the Fourier transform of $E\left(\overrightarrow{r_{\perp}}, t\right)$ :

$$
\widetilde{E}\left(\overrightarrow{r_{\perp}}, \omega\right)=\int E\left(\overrightarrow{r_{\perp}}, t\right) e^{i \omega t} d t
$$

It can be interesting to calculate the instantaneous power embedded in a particular mode $m$. According to Eq.7, the electric field $E_{m}\left(\overrightarrow{r_{\perp}}, t\right)$ in a mode $m$ writes:

$$
\begin{aligned}
E_{m}\left(\overrightarrow{r_{\perp}}, t\right) & =\int \bar{E}(m, \omega) \varepsilon\left(\overrightarrow{r_{\perp}}, t, m, \omega\right) d \omega \\
& =\int \bar{E}(m, \omega) \mathcal{F}\left(\overrightarrow{r_{\perp}}, m, \omega\right) e^{-i \omega t} d \omega .
\end{aligned}
$$

The optical power $P_{m}(t)$ in the mode $m$ is then given as:

$$
\begin{aligned}
P_{m}(t) & =\iint\left|E_{m}\left(\overrightarrow{r_{\perp}}, t\right)\right|^{2} r d r d \theta \\
& =\iint\left|T F^{-1}\left[\bar{E}(m, \omega) \mathcal{F}\left(\overrightarrow{r_{\perp}}, m, \omega\right)\right]\right|^{2} r d r d \theta .
\end{aligned}
$$

Accordingly, the optical power in the mode $m$ does not correspond to $|\bar{E}(m, t)|^{2}$ in the general case. In fact, the relation $P_{m}(t)=$ $|\bar{E}(m, t)|^{2}$ is valid only if one considers that the transversal shape of the mode $m$ does not depend on $\omega$, which is, strictly speaking, never the case in realistic waveguides.

Expressed in the modal basis, the unidirectional pulse propagation equation driving the evolution of the electric field $E$ along the coordinate $z$ is given by [18]:

$$
\begin{aligned}
\partial_{z} \bar{E}(m, \omega) & =i\left(\beta(m, \omega)-\frac{\omega}{v_{g_{0}}}\right) \bar{E}(m, \omega) \\
& +\frac{i \omega^{2}}{2 \epsilon_{0} c^{2} \beta(m, \omega)} \bar{P}_{\mathrm{NL}}(m, \omega),
\end{aligned}
$$

where $\beta(m, \omega)$ is the propagation constant of the $m^{\text {th }}$ mode at a given $\omega, \epsilon_{0}$ is the vacuum permittivity, $c$ is the light velocity in vacuum, and $\bar{P}_{\mathrm{NL}}(m, \omega)$ is the nonlinear polarization expressed in the modal basis. Moreover, for convenience, the equation is 
written in a local frame propagating at an arbitrarily chosen velocity $v_{g_{0}}$. The latter is often chosen as the group velocity of the fundamental mode $(m=1)$ calculated at the initial central frequency $\omega_{0}$ of the laser pulse.

In the context of fiber propagation, using a complex representation of the electric field $\xi$ (expressed now so that $|\xi|^{2}=I(r, t)$, $I$ being the pulse intensity), we obtain the MM-UPPE as follows:

$$
\begin{gathered}
\partial_{z} \bar{\zeta}(m, \omega)=i\left(\beta(m, \omega)-\frac{\omega}{v_{g_{0}}}\right) \bar{\xi}+\frac{i n_{\mathrm{eff}_{0}} n_{2} \omega^{2}}{c^{2} \beta(m, \omega)} \\
\left\{\left(1-f_{R}\right) \overline{|\xi|^{2} \xi}+f_{R} \overline{\left[\int h_{R}(\tau)|\xi(t-\tau)|^{2} d \tau\right] \xi}\right\},
\end{gathered}
$$

where $n_{\text {eff }}$ is the effective refractive index of the fundamental mode at the central frequency $\omega_{0}$ of the laser pulse, $n_{2}=\frac{3}{4} \frac{\chi^{(3)}}{\epsilon_{0} c n_{\mathrm{eff}_{0}}^{2}}$ is the nonlinear refractive index of the medium (here for silica glass, we used $n_{2}=3.2 \times 10^{-20} \mathrm{~m}^{2} / \mathrm{W}$ ) [20]. The function $h_{R}$ is the Raman response of the fiber medium, with Raman fraction $f_{R}=0.18$ for fused silica glass. We used an intermediatebroadening model using convolutions of Lorentzians and Gaussians adapted from spontaneous Raman scattering spectra and estimated Raman gain coefficient given in [32].

For the following, we will limit our discussion to the case where the refractive index only depends on $r$ (in cylindrical coordinates) and to cylindrically symmetric modes. As described in [18], solving this equation is performed in two distinct steps. First, for each $\omega$, the solver calculates the modes and its propagation constant. This is done in an efficient way by projecting the operator $\square_{\perp}$ on a basis composed of zeroth-order Bessel functions (i.e., decomposing any function of space coordinate $r$ as a zeroth-order Bessel-Fourier series). Using $N_{r}$ points in space, the diagonalization procedure will then give $N_{r}$ eigenvectors (i.e., modes) expressed in the Bessel basis (i.e., in the Hankel space) and their associated eigenvalues. Depending on their eigenvalues, these eigenvectors will correspond to either coreguided, clad-guided or evanescent modes. Calling $V_{\omega}$ the matrix filled with the eigenvectors coordinates, an electric field whose coordinates are $F$ in the Hankel space (i.e., in the Bessel basis) will write $F^{\prime}=V_{\omega} F$ in the modal basis. Numerically speaking, an electric field (at a given $\omega$ ) is evaluated at particular values of $r$, denoted $r_{j}$ (with $1 \leq j \leq N_{r}$ ), which forms a vector $F_{j}(\omega)$. In order to express the electric field in the Bessel basis, one has to calculate the Hankel transform of $F_{j}(\omega)$, which is performed by multiplying $F_{j}(\omega)$ by an unitary matrix $H: F=H F_{j}(\omega)$. As a consequence, in order to go from a spatial representation of the field to a modal one, one has:

$$
F^{\prime}(\omega)=M_{\omega} F_{j}(\omega),
$$

with $M_{\omega}=V_{\omega} H$. Reciprocally, one also has

$$
F_{j}(\omega)=M_{\omega}^{-1} F^{\prime}(\omega) .
$$

Using this fast modal transform then allows to solve the propagation very efficiently by a split-step algorithm during which the linear propagation term is evaluated within the modal basis while the nonlinear propagation step is calculated in the direct $(r, t)$ space.

\section{B. Multimode generalized nonlinear Schrödinger equations}

The derivation of the MM-GNLSE from Maxwell's equations or UPPE can be found in $[8,10]$. One has to emphasize that during the derivation of MM-GNLSE, the space and frequency dependence of a given mode is assumed as decoupled. Consequently, a mode is assumed to have the same transverse distribution whatever the frequency is. While this assumption is fair for relatively narrow spectra, it can lead to unphysical situation in the case of supercontinua or few-cycle pulse propagation simulations. In particular, in the case of multimode fibers, this assumption leads to say that the same number of modes is guided whatever the frequency is, while it is well known that high frequency supports more guided modes than low frequencies (by contrast, the MM-UPPE is not restricted to the modes that are guided within the fiber core but remains exact for modes that are guided within the clad or for evanescent modes). Here the frequency dependence of the resulting transverse overlap integrals between guided modes is truncated to the linear term around $\omega_{0}$. The MM-GNLSE is given by the following set of coupled equations to analyze the evolution of electric field amplitude envelope of an optical pulse as it propagates through multimode fibers

$$
\begin{aligned}
& \partial_{\zeta} A_{m}(\zeta, \tau)=D\left(A_{m}\right)+i \frac{n_{2} \omega_{0}}{\mathrm{c}}\left(1+\frac{i}{\omega_{0}} \partial \tau\right) \\
& \times \sum_{l, p, n}^{N}\left[\left(1-f_{R}\right) S_{m l p n}^{K} A_{l} A_{p} A_{n}^{*}+\right. \\
& \left.+f_{R} S_{m l p n}^{R} A_{l} \times \int_{-\infty}^{\tau}\left[h_{R}\left(\tau^{\prime}\right) A_{p}\left(z, \tau-\tau^{\prime}\right) A_{n}^{*}\left(z, \tau-\tau^{\prime}\right)\right] \mathrm{d} \tau^{\prime}\right]
\end{aligned}
$$

where $A_{m}(\zeta, \tau)$ is the complex temporal envelope of the $m^{\text {th }}$ mode. This approach allows to investigate observed phenomena in division modes. Additionally by switching on/off terms corresponding to some exact processes their real participation in the inspected effect could be examined.

The first right-hand side term in Eq. (16): $D\left(A_{m}\right)$ stands for the dispersion operator in time domain, which could be expressed as follows with a Taylor expansion:

$$
\begin{aligned}
D\left(A_{m}\right)= & i\left(\beta_{0}\left(m, \omega_{0}\right)-\operatorname{Re}\left[\beta_{0}\left(1, \omega_{0}\right)\right]\right) A_{m} \\
& -\left(\beta_{1}\left(m, \omega_{0}\right)-\operatorname{Re}\left[\beta_{1}\left(1, \omega_{0}\right)\right]\right) \partial_{\tau} A_{m} \\
& +i \sum_{j \geq 2} \frac{\beta_{j}\left(m, \omega_{0}\right)}{j !}\left(i \partial_{\tau}\right)^{j} A_{m},
\end{aligned}
$$

where $\beta_{j}=\partial_{\omega}^{j} \beta(m, \omega)$. In our simulations, we used the full $\beta(m, \omega)$ dependencies obtained with the scalar mode solver. The dispersion operator of $m^{\text {th }}$ mode expressed in frequency domain takes the following form:

$$
\widetilde{D}\left(\widetilde{A}_{m}\right)=i\left(\beta(m, \omega)-\beta_{0}\left(1, \omega_{0}\right)-\beta_{1}\left(1, \omega_{0}\right) \Omega\right) \widetilde{A}_{m}
$$

where $\widetilde{A}_{m}$ is a spectral amplitude of $m^{\text {th }}$ mode obtained as a Fourier transform of $A_{m}, \Omega=\left(\omega-\omega_{0}\right)$, and $\omega_{0}=2 \pi c / \lambda_{0}$.

Next terms in Eq. (16) represent the effects of optical nonlinearity with the nonlinear refractive index $n_{2} . S_{m l p n}^{K}$ and $S_{m l p n}^{R}$ are the mode coupling tensors for the Raman and Kerr effect respectively [9]. For linearly polarized modes we have

$$
\begin{aligned}
S_{m l p n}^{K} & =S_{m l p n}^{R}= \\
& \frac{\int\left[F_{m} F_{l} F_{p} F_{n}\right] \mathrm{d} x \mathrm{dy}}{\sqrt{\int F_{m}^{2} \mathrm{~d} x \mathrm{~d} y \int F_{l}^{2} \mathrm{~d} x \mathrm{~d} y \int F_{p}^{2} \mathrm{~d} x \mathrm{~d} y \int F_{n}^{2} \mathrm{~d} x \mathrm{~d} y}},
\end{aligned}
$$

where $F_{m}$ is the electric field of $m^{\text {th }}$ mode calculated with a scalar mode solver at $\omega_{0}$. It is worth noting, that $S_{m m m m}$ equals the inverse of the effective mode area of $m^{\text {th }}$ mode, $S_{m m m m}=1 / A_{\mathrm{eff}}^{(m)}$. 

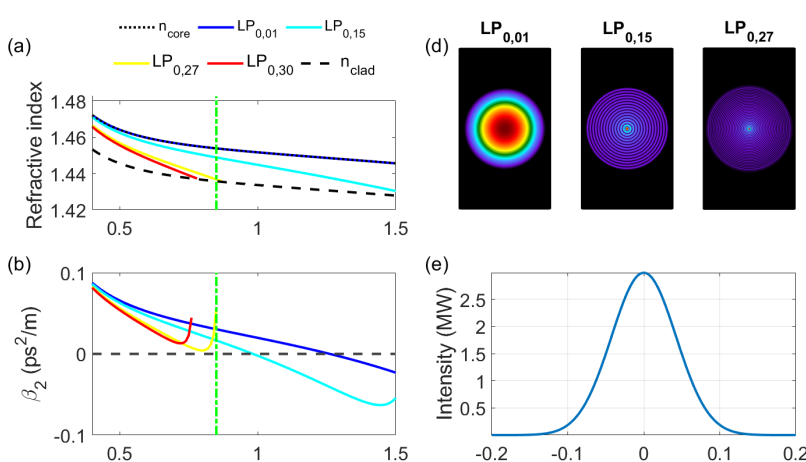

(c)
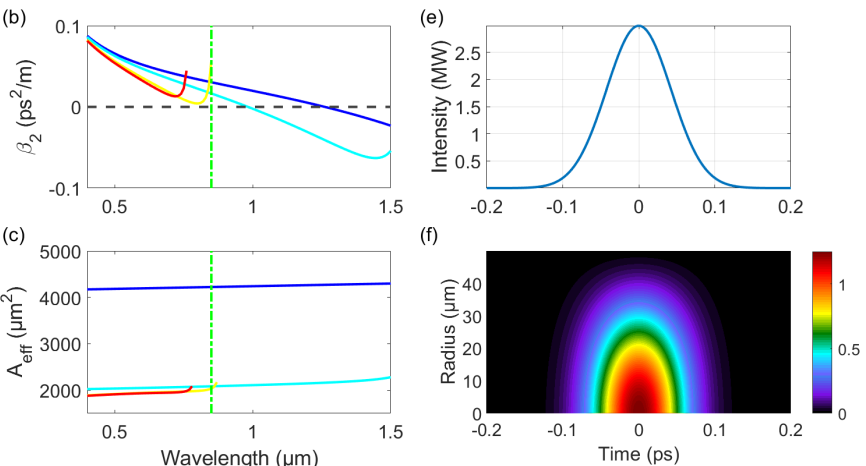

Fig. 1. Parameters of fiber and input pulse. (a) Refractive indices of the core (solid black), the clad (dashed black), and exemplary $\mathrm{LP}_{0, \mathrm{n}}$ modes of the optical fiber, (b) associated group velocity dispersion, and (c) associated effective mode areas as a function of wavelength. Dashed green lines indicate the central pump wavelength. (d) Normalized electric field of exemplary modes and (e) power of input pulse as a function of time (f) intensity of input pulse as a function of time and radius at the beginning of the fiber.

In our simulations of MM-GNLSE model, we used the solver architecture developed by Wright [3]. The approach is computationally demanding, but the calculations can be paralleled with massively parallel algorithm (MPA) [33]. Here, we apply the solver implementing MPA, which was developed by Wright et al. [11].

\section{Fiber properties}

In the following, we first consider a commercially-available multimode step-index fiber with a pure silica glass core (Thorlabs, FG105LCA). Fiber parameters were calculated according to the information provided by the manufacturer, namely the core diameter $(\Phi=105 \mu \mathrm{m})$ and the numerical aperture $(\mathrm{NA}=0.22 \pm 0.02)$. In our modal calculations, we account the material dispersion of pure silica glass as given by the Sellmeier formula from Ref. [34]. We then fixed the difference of refractive indices of core and cladding glasses to an approximated fixed value $\Delta n=0.01675$. The refractive indices of core and cladding glasses are shown in Fig. 1(a). Note that fiber losses (less than $10 \mathrm{~dB} / \mathrm{km}$ for the wavelength range under study) can be taken into account in our modelings, however their impact was fully negligible on the few-cm-long segment studied.

The described step-index fiber supports at $\lambda_{0}=850 \mathrm{~nm}$ more than 3600 modes. The number of modes $M$ can be easily calculated as $M=V^{2} / 2$ [35], where $V$ is a normalized frequency

$$
V=\frac{2 \pi}{\lambda_{0}} \frac{\Phi}{2} \sqrt{n_{\text {core }}^{2}-n_{\text {clad }}^{2}} .
$$

We developed the scalar mode solver based on the well-known Hondros-Debye equation for circular symmetry waveguides to calculate effective refractive indices and electric field distributions of the guided modes [35]. The mode solver allow us to calculate the properties of all guided modes. However, it would be practically impossible to account all of them in simulations of nonlinear propagation.

We restricted ourselves to the linearly polarized modes of $\mathrm{LP}_{0, n}$ class, due to assumed excitation as will be described further. The fiber supports $27 \mathrm{LP}_{0, n}$ modes at chosen pump wavelength $\lambda_{0}=850 \mathrm{~nm}$. The wavelength dependency of effective refractive indices of a few chosen modes are presented in Fig. 1(a) with reference to material refractive indices of the core and cladding glasses. One can then calculate the group-velocity dispersion expressed as $\beta_{2}=\partial^{2} \beta / \partial \omega^{2}$, where $\beta$ is the propagation constant of the considered mode, shown in Fig. 1(b). The calculated distribution of the normalized electric field of exemplary modes at $\lambda_{0}$ is provided in Fig. 1(d). Knowing electric field distributions, we calculated wavelength dependency of the effective mode area $A_{\text {eff }}$, shown in Fig. 1(c). It is worth noting that effective area for high-order $\mathrm{LP}_{0, n}$ modes decreases with mode order.

\section{Numerical parameters}

The duration and efficiency of simulations with both models depend on different numerical parameters. We keep them aligned between models (whenever it was possible).

The MM-GNLSE calculations start with the mode solver. By solving scalar wave equation for wavelengths in $0.4 \mu \mathrm{m}-3.0 \mu \mathrm{m}$ range, we calculated the effective refractive indices and later the dispersion operator given by Eq. (18). We also used the electric field distributions at $\lambda_{0}$ to calculate the coupling coefficients according to equation (19). In the MM-GNLSE, we accounted the $15 \mathrm{LP}_{0, n}$ lowest orders modes: from $\mathrm{LP}_{0,1}$ to $\mathrm{LP}_{0,15}$. This gives us the $S_{m l p n}^{R}$ tensor with $15^{4}=50625$ elements. The accounted number of modes was limited by the size of memory (8 GB) on a graphic card used for parallelization.

In the case of MM-UPPE simulations, we limited the space coordinate to a radius $R=75 \mu \mathrm{m}$ with $N_{R}=50$ discretization points. The mode calculation returns $N_{R}$ eigenvalues for each wavelength [18], and can be divided into three categories, namely the guided modes, the propagative clad modes and evanescent modes (a maximum of 35 guided modes for short wavelengths was obtained for the above fiber). All the fiber modes are reconstructed by summing the Bessel functions composing the basis weighted by the coordinate of the mode on the basis. Effective refractive index experienced by the mode can be determined as well as group-velocity dispersion and effective area if needed. Corresponding results are not shown here, since it would be almost indistinguishable from previous ones for guided modes in MM-GNLSE.

In both models, we solve initial value problem over propagation distance with fixed step size $(\Delta z=10 \mu \mathrm{m})$. The step size should be small enough to resolve intermodal beat length [11] which is in the considered fiber $178 \mu \mathrm{m}$ at $0.85 \mu \mathrm{m}$ and $117 \mu \mathrm{m}$ at $1.3 \mu \mathrm{m}$ between $\mathrm{LP}_{0,1}$ and $\mathrm{LP}_{0,15}$. In MM-GNLSE solver, the calculations are paralleled over 20 sub-steps. The time grids were the same and given by width $T=10 \mathrm{ps}$ and number of equally distributed points $N_{T}=2^{13}$. Consequently, the frequency grids were also identical. In MM-GNLSE the number of points in a frequency grid determines the complexity of Fast Fourier Transform used to switch between time and frequency domains (independently in each considered mode). In MMUPPE the number of frequency points $\left(N_{\omega}=N_{T}\right)$ with the number of discretization points along radius $N_{R}$ determines the dimensions of the modal-frequency domain. 
As mentioned earlier, the modal properties are calculated in both models before the nonlinear propagation is simulated. In case of MM-GNLSE, any method can be used to calculate propagation constants and mode profiles. For example, the mode solver can be based on finite element method to account arbitrary fiber's geometry. The mode profiles are used to calculate the overlap integrals tensor, which (in general) has $N_{M}^{4}$ elements, where $N_{M}$ is number of considered modes. In MM-UPPE the modal properties are calculated by diagonalization of $N_{R} \times N_{R}$ matrix for each frequency point in the basis composed by Bessel functions. The result of diagonalization is used to build the modal transform, the latter is performed in each simulation step to switch between the modal-angular frequency domain and the space-time domain.

\section{E. Pumping conditions}

The pump pulse is fixed at $\lambda_{0}=850 \mathrm{~nm}$ (operating in the normal dispersion regime as shown in Fig. 1(b)), and we assume a gaussian pulse with $T_{0}=T_{\mathrm{FWHM}} / 1.665$ and $T_{\mathrm{FWHM}}=100 \mathrm{fs}$. The pumping configuration is a beam coupling into the fundamental mode of the fiber, namely $L P_{0,1}$. As a considered fiber is circularly symmetric, the coupling coefficients are nonzero only for $L P_{0, n}$ modes [9].

We investigate ultrashort pulse propagation in MMFs with a peak power around the critical power for self-focusing to evidence clear spatiotemporal nonlinear effects. For instance, nonlinear dynamics of femtosecond pulses undergoing self-focusing has been already studied experimentally with input powers up to four times the critical power in BK7 glass [27]. It is worth to note that the corresponding incident intensity is below the damage threshold of the dielectric material [36], but considerable ionization preceding the damage thresholds may take place for intensities beyond $10^{13} \mathrm{~W} / \mathrm{cm}^{2}[37,38]$. However, we favor the normal dispersion regime for which dispersion is known to dominate plasma generation in halting the collapse at powers far above critical power [39]. The critical self-focusing power is given as [24]

$$
P_{\text {crit }}=1.86 \frac{\lambda_{0}^{2}}{4 \pi n_{0} n_{2}}
$$

where $\lambda_{0}=850 \mathrm{~nm}, n_{0}=1.4525$ (refractive index for fundamental mode). We obtain $P_{\text {crit }}=2.3 \mathrm{MW}$. In the next section, the peak power was set to $P_{\mathrm{p}}=1.3 P_{\text {crit }}$ since the large normal dispersion is known to increase the self-focusing threshold [40]. Corresponding spatiotemporal initial pulse profiles are shown in Fig. 1(e) and Fig. 1(f).

Finally, in both models, noise effects during pulse propagation can be also studied through the addition of a noise seed in initial pumping pulse. In case of MM-GNLSE, we added noise to all considered modes with one-photon per mode with random phase on each spectral discretization bin [19]. For MM-UPPE, we simply used a white noise with random phase added to full field in the space-time domain. Note that the presence of noise does not modify the results described in the following.

\section{NUMERICAL RESULTS AND COMPARISON}

As a a first test, we compared maximum pulse intensities calculated with both modelings on short propagation distance $(1 \mathrm{~mm})$. Discrepancies appear negligible in such cases. Moreover we also evaluate reliability of our simulations by checking the conservation of photon number and total energy along propagation distance for both approaches. Figure 2 presents the numerical results obtained from both models MM-GNLSE and MM-UPPE
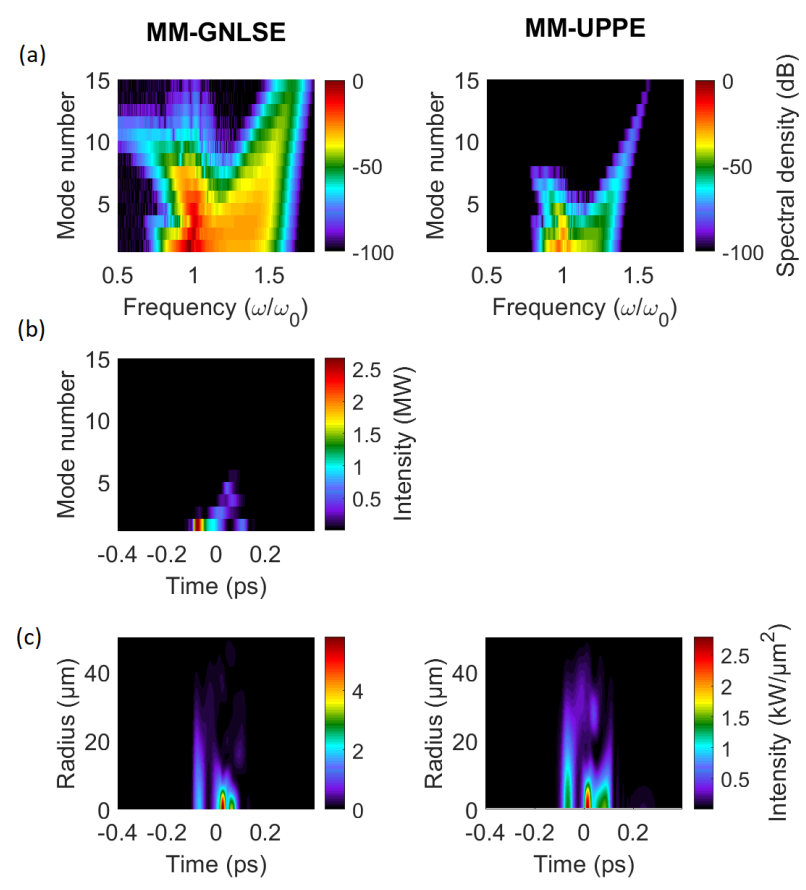

Fig. 2. Comparison of two numerical models for simulation parameters: $\lambda_{0}=850 \mathrm{~nm}, P_{0}=1.3 \times P_{\text {crit }}, \Phi=105 \mu \mathrm{m}$, $z=2 \mathrm{~cm}$. The results were presented using graphs commonly used for the discussed methods. (a) spectral energy density for distinct modes, (b) pulse intensity for distinct modes, (c) pulse intensity in space.

after $2 \mathrm{~cm}$ of propagation in the multimode fiber described above. In the case of MM-GNLSE (left column), the electric field is expressed as a combination of electric fields of a limited number of guided modes (15 in the case under study). The time-dependent power profiles is then known for each mode separately Fig. 2(a)), and it can be transformed into spectral dependency with one dimensional Fourier transform (Fig. 2(b)). The full electric field in the space-time domain could be restored by summing up contributions from all modes. This way, we calculate optical intensity as a function of radius and time (Fig. 2(c)). By contrast, in MM-UPPE approach (right column), the electric field is expressed explicitly in the space-time domain (Fig. 2(c)), and with both the Fourier transform and the fast modal transform, it can be expressed into the corresponding modal basis as a function of angular frequency (see Fig. 2(a)). The time-dependent modal power distribution can be evaluate by calculating $\bar{\xi}(m, t)$, even if one has to remind that the latter slightly deviates from the optical power embedded in one particular mode because of the frequency dispersion of the modal transversal shape, as discussed in Section 2.A. Note that, in MM-GNLSE, the approximation of frequency-invariant modal transversal shape is inherently made.

In both cases, we clearly observe a qualitative agreement about the strong spectral broadening (i.e., an octave spanning supercontinuum) associated with temporal compression of the pump pulse as well as energy spreading in higher-order modes from the fundamental mode. We note a typical signature of $X-$ pattern formation, namely that the lowest and highest frequencies generated are continuously contained in the fiber modes with increasing order up to the $15^{\text {th }}$. A detailed analysis of the phase-matching condition is provided in the next section. 
However, one can note significant discrepancies about the supercontinuum bandwidth, the power distribution between modes, as well as the temporal pulse splitting. More particularly, it appears that MM-GNLSE overestimates the overall nonlinear response compared to the MM-UPPE approach, thus leading to a larger spectrum, a higher peak power in the space-time domain, and larger power spectral densities in higher-order modes. In that respect, recall that mode coupling tensors $S_{m l p n}$ (Eq. (19)) are calculated only at the central pump wavelength $\lambda_{0}$ $[10,15]$. It means that the MM-GNLSE model does not take into account the frequency dependence of nonlinear effects unlike the MM-UPPE. In other words, the MM-GNLSE model only considers a simplified self-steepening term (also called sometimes shock term), which here comes from the Taylor expansion of the nonlinear coefficient of the fiber, whereas this nonlinear term in MM-UPPE is exact [22].

To give a better overview of the nonlinear dynamics during propagation, Figure 3 compares the longitudinal evolution of the spectral broadening, the pulse splitting, and the spatial behaviour over a longer propagation distance. As we study here the region of normal dispersion, we globally note a typical scenario that has been already analyzed in bulk dielectric media, namely that the self-focusing dynamics and spectral broadening are accompanied by pulse splitting phenomena [27]. The later is also known to arrest the collapse of high-power ultrashort pulses.

More particularly, a qualitative agreement is again noticeable through the asymmetry between the powers of the split subpulses, and the close link between pulse splitting and supercontinuum generation. To go into detail [41], self-phase modulation first broadens the pulse spectrum. The induced-nonlinear chirp implies that red-shifted and blue-shifted frequencies are generated at the leading and trailing edges of the pulse, respectively. Pulse splitting at the nonlinear focus produces two sub-pulses moving with opposite directions in our retarded time frame. The velocity difference between a pulse peak and its tails then induces optical shocks due to the nonlinear dependence of the refractive index. The slopes of dashed white lines in Fig. 3(b) indicate the relative group velocities of the shock front formed at the trailing edge. Pulse splitting is followed by a strong spectral broadening induced by the latter self-steepening effect.

The detailed evolution of spectral broadening near the pulse splitting process (i.e., about $1 \mathrm{~cm}$ of propagation) is shown in Fig. 4. We again retrieve the usual asymmetry of the supercontinuum related to the steep edge formed at the trailing edge of the trailing sub-pulse, giving rise to a strong blue-shifted pedestal in the spectrum [41]. Besides, we note that spectral broadening rapidly saturates after $2 \mathrm{~cm}$ of propagation only. This is associated to a significant and continuous temporal broadening of the sub-pulses as well as small periodic oscillations of the beam dimension after the main nonlinear focus.

Concerning the numerical differences between both models, Fig. 3 and Fig. 4 clearly show that MM-GNLSE overestimates the nonlinear response since the self-focusing and self-steepening effects appear to be faster and stronger in the space-time domain, thus leading to a larger supercontinuum bandwidth.

Finally, we compare the duration time of simulations (corresponding to Fig. 3) with both models performed at the same workstation gathered in Table 1 . In both cases the duration of the mode solver and nonlinear propagation are given separately. As described in section 2.D, the mode solver calculations for MMGNLSE include calculations of propagation constants as well as the complex overlap tensor, whereas only propagation con-
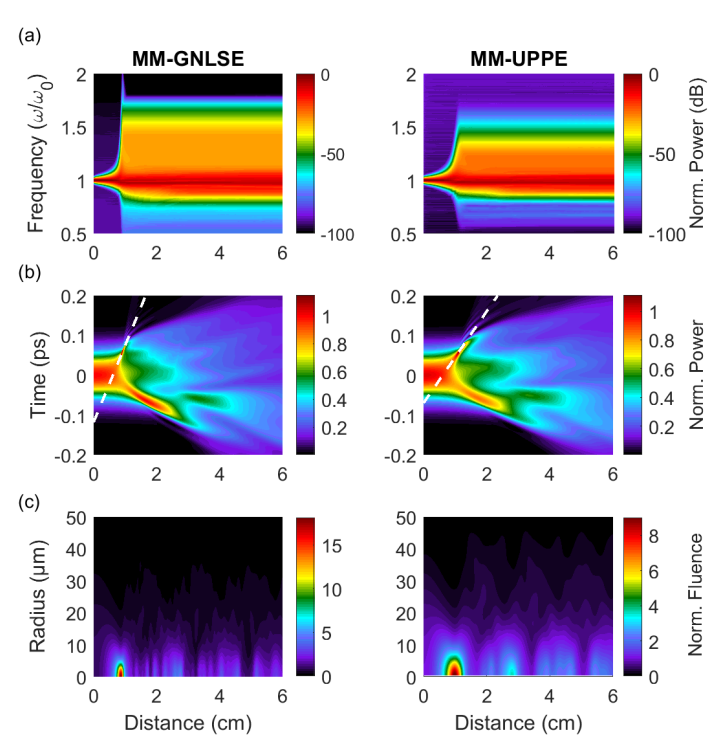

Fig. 3. (a) Evolution of the normalized integrated spectrum, (b) the normalized instantaneous power, and (c) the normalized fluence along the propagation distance in a $6 \mathrm{~cm}$ step-index fiber. The dashed white lines indicate the relative group velocity of shock front formed at the trailing edge of the pulse.
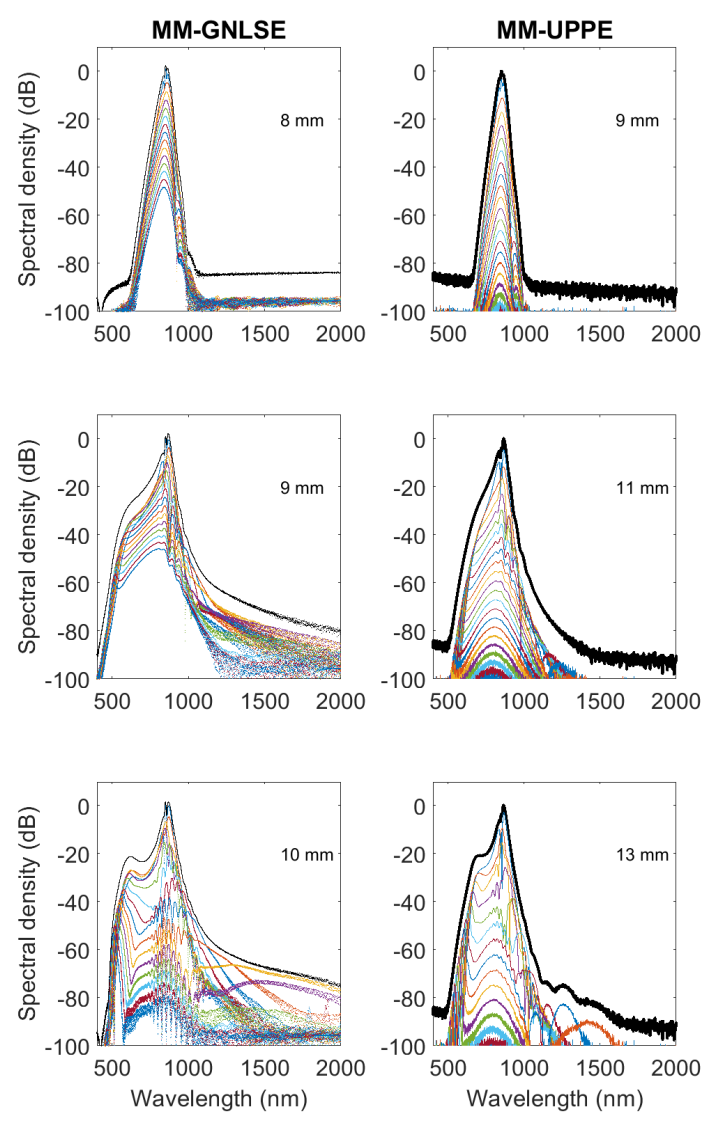

Fig. 4. Spectral profiles for each mode (color lines) and for its summation (black line) at 3 propagation distances: before, at exact point of formation of conical wave, and when the Xshape conical wave was established. Distances were almost the same for both numerical models. 
Table 1. Comparison of simulation times corresponding to Fig. 3 for both solvers on the same workstation.

\begin{tabular}{|c|c|c|}
\cline { 2 - 3 } \multicolumn{1}{c|}{} & MM-GNLSE & MM-UPPE \\
\hline mode solver & 18 minutes & 0.3 minute \\
\hline nonlinear propagation & 3.4 hours & 0.75 hour \\
\hline
\end{tabular}

stants and the fast modal transform are calculated in MM-UPPE. Note that the MM-GNLSE solver implements massively parallel algorithm (MPA) and is performed on GPUs. By contrast, MMUPPE solver operates on CPU. The workstation operates under Windows 10 operating system on Intel Core i9 9900K CPU with 32 GBytes DDR4 memory and uses NVIDIA GeForce RTX 2080 graphic card.

As already mentioned, our simulations clearly show that space-time focusing of ultrashort pulses implies a particular modal distribution of newly generated frequency components, namely an X-shape pattern observed in both the modal-angular frequency domain. In other words, we show that the axial supercontinuum generation is also connected with colored conical emission [41-43]. Based on previous studies of filamentation in various experimental conditions, the $X$ shape in the Fourier domain usually follows the linear dispersion characteristics of the medium which determines the relation between the propagation angle and frequency for phase-matched off-axis conical emission. This is the spectral counterpart of space-time coupling. In the following, we make use of the recent generalization of conical wave formation in structured media, such as multimode optical fibers [44], to analyze their observation in both numerical modelings.

\section{CONICAL EMISSION}

Conical emission has been intensively studied in dispersive nonlinear bulk media, in particular in the filamentation regime [26]. Only recently, the possibility to generate such kind of waves in fibers was suggested in few works $[18,45]$. The interpretation of light filaments in the normal dispersion usually present typical features such as pulse splitting and conical emission, and it can be interpreted assuming the pulses as spontaneously emerging nonlinear X-waves [46, 47]. More specifically, off-axis (conical) tails emerge on both the blue and red-shifted sides of the input wavelength, forming a universal distinct X-shaped pattern of conical emission. It was found that the input pulse can evolve toward a final stationary state that has the form of either an $\mathrm{X}$-wave in the range of normal dispersion or an $\mathrm{O}$-wave in the range of anomalous dispersion [48]. Nonlinear $\mathrm{X}$-waves and $\mathrm{O}$-waves are named because of their evident $\mathrm{X}$-like and O-like shapes, respectively, which appear in both the near and the far fields [49]. The sign of dispersion governs the dynamics of femtosecond filaments and so the time-frequency content of the associated supercontinuum. It was demonstrated that the dispersion landscape is the key ingredient for accurately modeling conical emission as it governs the specific shape of the conical emission patterns (X-shaped for normal dispersion, O-shaped for anomalous dispersion, or fish-shaped as their combination when pumping close to the zero dispersion).

Here we investigate in detail the characteristics of the conical emission observed in both models for the multimode fiber under study. Figure 5 depicts the evolution of the conical emission as a function of propagation distance for both space-time and modal-frequency domains. The MM-UPPE simulation clearly indicates the pulse splitting phenomenon in space-time after $8 \mathrm{~mm}$ of propagation and a moderate spectral broadening. The two sub-pulses move apart in the retarded time frame, and an optical shock forms on the trailing pulse associated with significant spectral broadening. This process then seeds higher order modes as shown after $11 \mathrm{~mm}$ of propagation, thus inducing the emergence of $X$-features in the spectral domain. The insets in Fig. 5(b) show the spectrally filtered data when the main pulsed pump is removed. They confirm that the tail exhibiting an increasing mode-number with frequency detuning from the pump is departing from the trailing egde of the pulse in the time domain. Next we clearly note that well-defined frequencies for each mode constitute this tail with further propagation. Those frequencies correspond to the phase-matched frequencies of a discretized conical wave induced nonlinearly as described in Ref.[44].

Indeed the corresponding phase-matching condition of this conical wave relies on phase-matched resonant radiations (i.e., dispersive waves) seeded by the optical shock. The phasematched frequencies $\omega_{C}$ in $m^{\text {th }}$ mode fulfills the following relation (for both models, MM-UPPE and MM-GNLSE)

$\beta(m, \omega)-\left[\beta_{0}\left(1, \omega_{0}\right)+\delta \beta_{0}\right]-\left[\beta_{1}\left(1, \omega_{0}\right)+\delta \beta_{1}\right]\left[\omega_{C}-\omega_{0}\right]=0$.

Parameters $\delta \beta_{0}$ and $\delta \beta_{1}$ can be considered as corrections to the phase and group velocities at $\omega_{0}$, and related to the nonlinear dynamics, respectively. The parameter $\delta \beta_{0}$ governs the bandwidth of the phase-matching around the central frequency $\omega_{C}$ for each spatial mode, and the parameter $\delta \beta_{1}$ drives the group velocity of the conical wave governed by to the group velocity difference of the shock front in the retarded time frame [44]. As a result, the shape of the conical emission is mainly driven by the modal refractive index curves as well as the value of $\delta \beta_{1}$. From Fig. 3(b) we estimated the group velocity difference to $\delta \beta_{1} \simeq-12 \mathrm{ps} / \mathrm{m}$ (for MM-UPPE model), this allowed us to provide an accurate description based on Eq. 22 of the conical wave observed numerically, namely the two asymmetric discrete branches of (i.e., the discretized X-wave) in the mode number-frequency space separated by a gap in frequency (see white squares in Fig. 5(c)). Here $\delta \beta_{0}$ was first fixed to 0 , and then treated as a free parameter to describe the shape of the low-value contours of X-shaped pattern.

Similar analysis performed with MM-GNLSE results (Fig. 3(b)) requires a higher value of coefficient $\delta \beta_{1}$ since we observed a faster optical shock induced by a reduced dispersion of the nonlinearity with MM-GNLSE modeling. The group velocity difference here reaches $\delta \beta_{1} \simeq-19 \mathrm{ps} \mathrm{m}^{-1}$. Additionally, the pulse splitting and conical emission occur at smaller propagation distances than in MM-UPPPE modelling. As mentioned earlier, the MM-GNLSE overestimates the overall nonlinear response in comparison with MM-UPPE due to neglecting wavelength dependency of the modes coupling tensor. However, we found that the frequencies obtained from phase-matching condition agree with mode number resolved spectra as shown in Fig. 6(c). The MM-GNLSE allowed us to inspect also modal-time field distribution (middle row in Fig. 6). The results of MM-GNLSE simulations confirm that the energy conversion to higher frequencies takes place at the trailing edge of the pulse and only for specific phase-matched frequency of each mode. Finally, we easily observe that the cone emitted at the shock front position propagates into the fiber in a dispersive manner as theoretically predicted in Ref. [44]. 

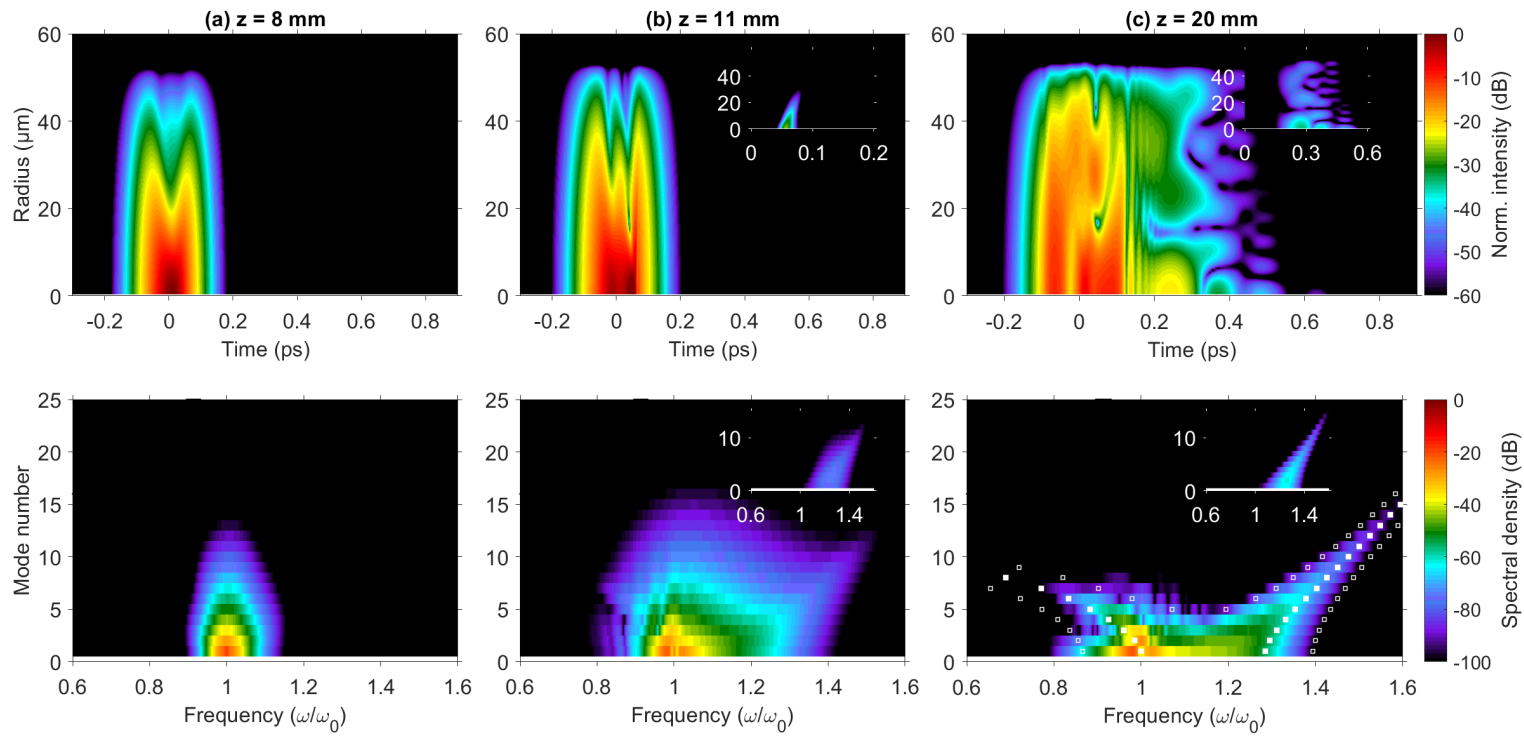

Fig. 5. Evolution of conical emission as a function of propagation distance obtained from MM-UPPE. The intensity distributions in the space-time domain are shown in the top row. The spectral energy density in the spatial modes are shown in bottom row. Data are presented at three propagation distances: (a) $z=8 \mathrm{~mm},(\mathrm{~b}) z=11 \mathrm{~mm}$, (c) $z=20 \mathrm{~mm}$. The insets show spectrally filtered data revealing the conical emission at the trailing edge of the pulse. The white squares in (c)-bottom indicate central phase-matched frequencies in each spatial mode (as well as phase-matching bandwidth).

(a) $z=7 \mathrm{~mm}$
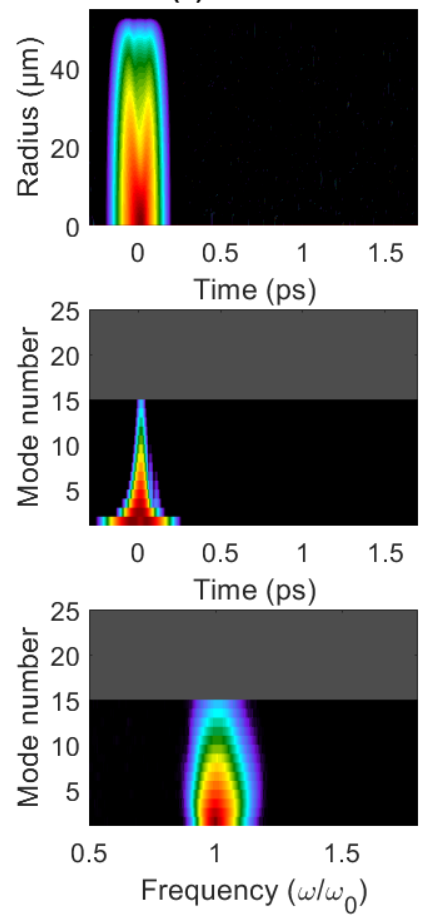

(b) $z=9 \mathrm{~mm}$
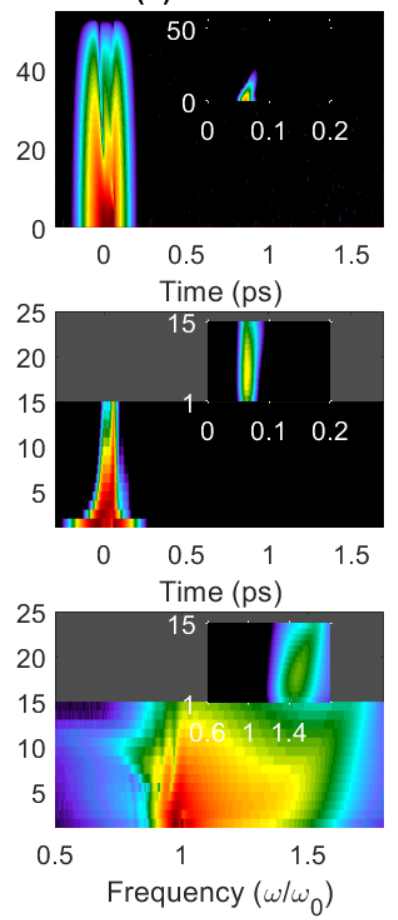

(b) $z=20 \mathrm{~mm}$
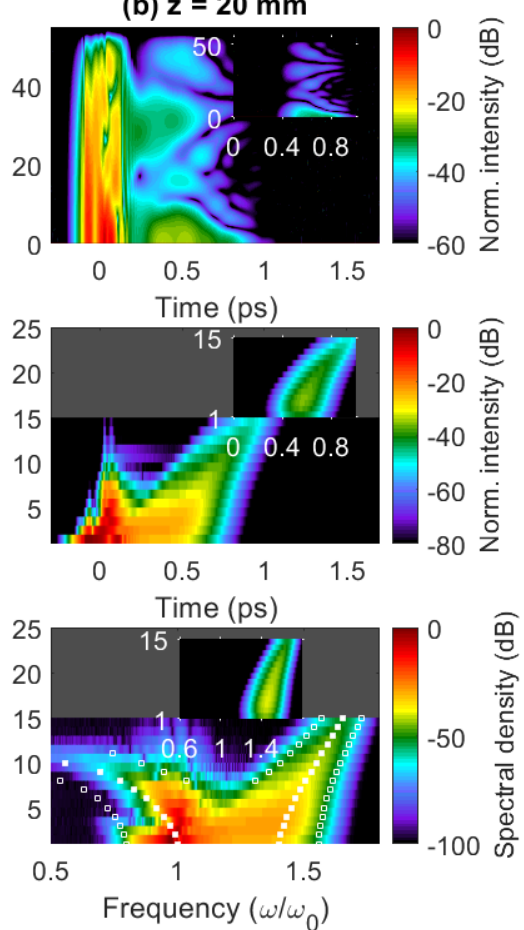

Fig. 6. Evolution of conical emission as a function of propagation distance obtained from MM-GNLSE. The intensity distributions in the space-time domain are shown in the top row. The intensity distributions in the spatial modes are shown in middle row. The spectral energy density in spatial modes are shown in bottom row. Data are presented at three propagation distances: (a) $z=7 \mathrm{~mm}$, (b) $z=9 \mathrm{~mm}$, (c) $z=20 \mathrm{~mm}$. The insets show spectrally filtered data revealing the conical emission at the trailing edge of the pulse. The white squares in (c)-bottom indicate central phase-matched frequencies in each spatial mode (as well as phase-matching bandwidth). The grey regions in middle and bottom rows indicate the spatial modes which are not considered in MM-GNLSE approach. 
For the sake of completeness, we also performed numerical simulations with both modelings for smaller and bigger fiber cores, namely $\Phi=50 \mu \mathrm{m}$ and $\Phi=200 \mu \mathrm{m}$. Note again that such dimensions are similar to commercially-available stepindex multimode fibers (Thorlabs FG050LGA and FG0200LGA). The presented results from MM-UPPE in Fig. 7 could be refereed to measurements, which would be performed in future experiments, since they could be easily described through the expected angle-resolved conical emission spectra. Note that an increasing input power was required to observe the conical emission when extending the core diameter, (i.e., when increasing the effective mode area of the excited fundamental mode). For our studies, we used $P_{\mathrm{p}}=1.1 P_{\text {crit }}$ and $P_{\mathrm{p}}=1.8 P_{\text {crit }}$ for $\Phi=50 \mu \mathrm{m}$ and $\Phi=200 \mu \mathrm{m}$, respectively. Other input parameters remained unchanged. It is worth to mention that a less intense optical shock on the leading edge of the pump pulse gives rise to another discretized X-wave, this phenomenon becomes noticeable when $\Phi=200 \mu \mathrm{m}$ (see Fig. 7(c), other tails can be observed).

Similar simulations were also performed by using the MMGNLSE modeling (not shown here). For $\Phi=50 \mu \mathrm{m}$, due to a smaller number of guided modes, we considered all the 13 modes of $\mathrm{LP}_{0, n}$ class supported at the pump wavelength. By contrast, for $\Phi=200 \mu \mathrm{m}$, only 15 modes were included over 52 at the pump wavelength, due to memory limitations. As expected, we can achieve better compatibility between models for smaller core size, which is closely related to the use of lower input power and numbers of accounted modes. A detailed investigation of the impact of spatial input excitation and fiber properties will be presented in future works.

\section{SUMMARY}

In this work we numerically studied the highly nonlinear regime of ultrashort pulse propagation (i.e., fs pulse with peak power around the self-focusing critical power of the medium) in multimode step-index silica fibers by comparing two well-known modeling approaches: (i) the MM-GNLSE is a common used tool to investigate propagation of light in multimode fibers, (ii) the use of unidirectional propagation equations (like MM-UPPE) is a more general approach rather used for bulk medium, and most of propagation equations in waveguides can be derived from it. As expected, the difference in the nonlinear step of modelings is the main origin of discrepancies. First, in the case of MM-GNLSE, the mode coupling tensors were calculated only at the central pump wavelength, which leads to non-dispersive effective mode areas, but more importantly in this model, the self-steepening term is expressed by approximation of the propagation constants with its 1st order Taylor expansion. Both approximations do not apply to the MM-UPPE approach. This mainly results in overestimating the full nonlinear response and associated spectral broadenings in the femtosecond regime. Our configuration appears as a good starting point to reveal some limitations of approximated numerical models such as the MM-GNLSE. However, our numerical studies have shown that outcomes from both models are qualitatively in good agreement. In particular, we clearly reveal typical spatio-temporal dynamics commonly observed from femtosecond pulse filamentation in bulk media with normal dispersion, such as a strong space-time reshaping followed by pulse splitting and conical emission. Our results confirm that the modal distribution of optical fibers provides a discretization of conical waves of the corresponding bulk medium [44].

In this work, we limited ourselves to pumping at single pump wavelength in normal dispersion regime, as our goal was to compare two modeling approaches. Further perspectives include considering pumping in different dispersion regimes to observe the formation of other types of conical waves as well as using soft-glass or engineered multimode fibers with various dispersion landscapes. In conclusion, future nonlinear experiments in commercially-available optical fibers could easily reveal novel alluring phenomena, such as the possible formation of supercontinuum light bullets in standard multimode fibers.

The project is co-financed by the Polish National Agency for Academic Exchange and French-Polish Polonium Hubert Curien Partnership 2019-2020. B.K. also acknowledges financial support of the French "Investissements d'Avenir" program (PIA2/ ISITEBFC,Contract ANR-15-IDEX-03, Project "Breathing Light"). K.T. and S.M. also acknowledges financial support of the National Science Center, Poland (project no. 2018/30/E/ST7/00862, SONATA BIS 8 Program). B.K. thanks Dr Gang Xu for stimulating discussions.

\section{DISCLOSURES}

The authors declare no conflicts of interest.

\section{REFERENCES}

1. A. Picozzi, G. Millot, and S. Wabnitz, "Nonlinear optics: Nonlinear virtues of multimode fibre," Nat. Photonics 9, 289-291 (2015).

2. K. Krupa, A. Tonello, A. Barthélémy, T. Mansuryan, V. Couderc, G. Millot, P. Grelu, D. Modotto, S. A. Babin, and S. Wabnitz, "Multimode nonlinear fiber optics, a spatiotemporal avenue," APL Photonics 4, 110901 (2019).

3. L. G. Wright, D. N. Christodoulides, and F. W. Wise, "Controllable spatiotemporal nonlinear effects in multimode fibres," Nat. Photonics 9, 306-310 (2015).

4. L. G. Wright, Z. Liu, D. A. Nolan, M. J. Li, D. N. Christodoulides, and F. W. Wise, "Self-organized instability in graded-index multimode fibres," Nat. Photonics 10, 771-776 (2016).

5. R. J. Essiambre, M. A. Mestre, R. Ryf, A. H. Gnauck, R. W. Tkach, A. R. Chraplyvy, Y. Sun, X. Jiang, and R. Lingle, "Experimental investigation of inter-modal four-wave mixing in few-mode fibers," IEEE Photonics Technol. Lett. 25, 539-542 (2013).

6. W. H. Renninger and F. W. Wise, "Optical solitons in graded-index multimode fibres," Nat. Commun. 4, 1716-1719 (2013).

7. W. Fu, L. G. Wright, P. Sidorenko, S. Backus, and F. W. Wise, "Several new directions for ultrafast fiber lasers," Opt. Express 26, 9432-9463 (2018).

8. M. Kolesik and J. V. Moloney, "Nonlinear optical pulse propagation simulation: From Maxwell's to unidirectional equations," Phys. Rev. E 70, 11 (2004).

9. P. Horak and F. Poletti, "Multimode nonlinear fibre optics: Theory and applications," in Recent Progress in Optical Fiber Research, M. Yasin, S. W. Harun, and H. Arof, eds. (IntechOpen, Rijeka, 2012), chap. 1.

10. F. Poletti and P. Horak, "Description of ultrashort pulse propagation in multimode optical fibers," J. Opt. Soc. Am. B 25, 1645-1653 (2008).

11. L. G. Wright, Z. M. Ziegler, P. M. Lushnikov, Z. Zhu, M. A. Eftekhar, D. N. Christodoulides, and F. W. Wise, "Multimode nonlinear fiber optics: Massively parallel numerical solver, tutorial, and outlook," IEEE J. Sel. Top. Quantum Electron. 24, 1-16 (2018).

12. J. T. Manassah, P. L. Baldeck, and R. R. Alfano, "Self-focusing and self-phase modulation in a parabolic graded-index optical fiber," Opt. Lett. 13, 589 (1988).

13. S. Longhi, "Modulational instability and space time dynamics in nonlinear parabolic-index optical fibers," Opt. Lett. 28, 2363 (2003).

14. K. Krupa, A. Tonello, A. Barthélémy, V. Couderc, B. M. Shalaby, A. Bendahmane, G. Millot, and S. Wabnitz, "Observation of Geometric Parametric Instability Induced by the Periodic Spatial Self-Imaging of Multimode Waves," Phys. Rev. Lett. 116, 183901 (2016). 

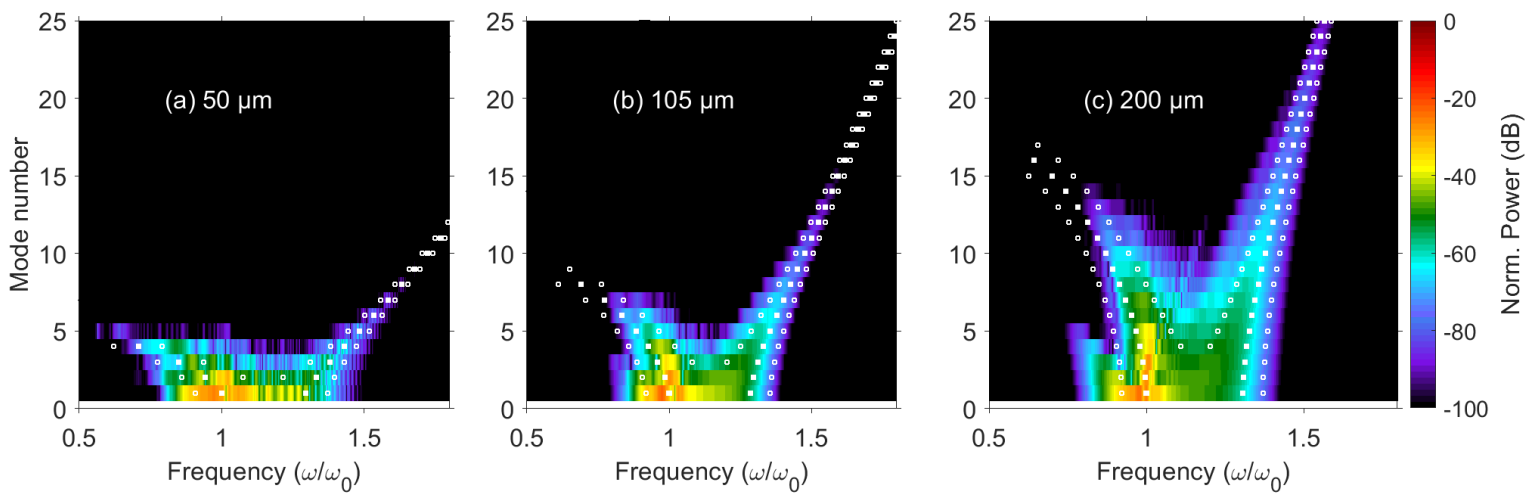

Fig. 7. Mode number-resolved conical emission spectra simulated after $6 \mathrm{~cm}$ of propagation in a multimode step-index fiber with increasing core diameters, namely $\Phi=50 \mu \mathrm{m}, \Phi=105 \mu \mathrm{m}$ and $\Phi=200 \mu \mathrm{m}$ obtained from MM-UPPE modeling. The white squares indicate central phase-matched frequencies in each spatial mode (as well as phase-matching bandwidth) according to equation (22).

15. J. Lægsgaard, "Efficient simulation of multimodal nonlinear propagation in step-index fibers," J. Opt. Soc. Am. B 34, 2266 (2017).

16. M. Conforti, C. Mas Arabi, A. Mussot, and A. Kudlinski, "Fast and accurate modeling of nonlinear pulse propagation in graded-index multimode fibers," Opt. Lett. 42, 4004 (2017).

17. J. Andreasen and M. Kolesik, "Nonlinear propagation of light in structured media: Generalized unidirectional pulse propagation equations," Phys. Rev. E - Stat. Nonlinear, Soft Matter Phys. 86, 1-9 (2012).

18. P. Béjot, "Multimodal unidirectional pulse propagation equation," Phys. Rev. E 99, 032217 (2019).

19. J. M. Dudley, G. Genty, and S. Coen, "Supercontinuum generation in photonic crystal fiber," Rev. Mod. Phys. 78, 1135-1184 (2006).

20. G. P. Agrawal, Nonlinear Fiber Optics, 5th Edition (Academic Press, 2012).

21. M. Kolesik, J. V. Moloney, and M. Mlejnek, "Unidirectional Optical Pulse Propagation Equation," Phys. Rev. Lett. 89, 283902 (2002).

22. A. Couairon, E. Brambilla, T. Corti, D. Majus, O. d. J. Ramirez-Gongora, and M. Kolesik, "Practitioner's guide to laser pulse propagation models and simulation," The Eur. Phys. J. Special Top. 199, 5-76 (2011).

23. A. D. Bandrauk, E. Lorin, and J. V. Moloney, Laser Filamentation, Mathematical Methods and Models (Springer, 2016).

24. A. Couairon and A. Mysyrowicz, "Femtosecond filamentation in transparent media," Phys. Reports 441, 47-189 (2007).

25. M. Kolesik, E. M. Wright, and J. V. Moloney, "Interpretation of the spectrally resolved far field of femtosecond pulses propagating in bulk nonlinear dispersive media," Opt. Express 13, 10729 (2005).

26. A. Couairon, D. Faccio, and P. D. Trapani, Conical Waves, Filaments and Nonlinear Filamentation Optics (Aracne, Rome, 2007).

27. J. K. Ranka, R. W. Schirmer, and A. L. Gaeta, "Observation of pulse splitting in nonlinear dispersive media," Phys. Rev. Lett. 77, 3783-3786 (1996).

28. Z. Sanjabi Eznaveh, M. A. Eftekhar, J. E. Antonio Lopez, M. Kolesik, A. Schülzgen, F. W. Wise, D. N. Christodoulides, and R. Amezcua Correa, "Tailoring frequency generation in uniform and concatenated multimode fibers," Opt. Lett. 42, 1015 (2017).

29. R. Dupiol, A. Bendahmane, K. Krupa, A. Tonello, M. Fabert, B. Kibler, T. Sylvestre, A. Barthelemy, V. Couderc, S. Wabnitz, and G. Millot, "Cascaded intermodal four-wave mixing in a few-mode fiber," Opt. Lett. 42, 1293-1296 (2017).

30. L. Rishøj, B. Tai, P. Kristensen, and S. Ramachandran, "Soliton selfmode conversion: revisiting Raman scattering of ultrashort pulses," Optica. 6, 304 (2019).

31. M. A. Eftekhar, Z. Sanjabi-Eznaveh, H. E. Lopez-Aviles, S. Benis, J. E. Antonio-Lopez, M. Kolesik, F. Wise, R. Amezcua-Correa, and D. N. Christodoulides, "Accelerated nonlinear interactions in graded-index multimode fibers," Nat. Commun. 10, 1638 (2019).
32. D. Hollenbeck and C. D. Cantrell, "Multiple-vibrational-mode model for fiber-optic raman gain spectrum and response function," J. Opt. Soc. Am. B 19, 2886-2892 (2002).

33. P. M. Lushnikov, "Fully parallel algorithm for simulating dispersionmanaged wavelength-division-multiplexed optical fiber systems," Opt. Lett. 27, 939 (2002).

34. H. R. Sunak and S. P. Bastien, "Refractive Index and Material Dispersion Interpolation of Doped Silica in the 0.6-1.8 $\mu \mathrm{m}$ Wavelength Region," IEEE Photonics Technol. Lett. 1, 142-145 (1989).

35. A. Snyder and J. Love, Optical Waveguide Theory (Springer, 1983).

36. B. Chimier, O. Utéza, N. Sanner, M. Sentis, T. Itina, P. Lassonde, F. Légaré, F. Vidal, and J. C. Kieffer, "Damage and ablation thresholds of fused-silica in femtosecond regime," Phys. Rev. B - Condens. Matter Mater. Phys. 84, 094104 (2011).

37. D. M. Rayner, A. Naumov, and P. B. Corkum, "Ultrashort pulse nonlinear optical absorption in transparent media," Opt. Express 13, 3208 (2005).

38. A. Couairon, L. Sudrie, M. Franco, B. Prade, and A. Mysyrowicz, "Filamentation and damage in fused silica induced by tightly focused femtosecond laser pulses," Phys. Rev. B - Condens. Matter Mater. Phys. 71, 1-11 (2005).

39. S. Skupin and L. Bergé, "Self-guiding of femtosecond light pulses in condensed media: Plasma generation versus chromatic dispersion," Phys. D: Nonlinear Phenom. 220, 14-30 (2006).

40. G. G. Luther, E. M. Wright, J. V. Moloney, and A. C. Newell, "Selffocusing threshold in normally dispersive media," Opt. Lett. 19, 862 (1994).

41. A. Dubietis and A. Couairon, Ultrafast Supercontinuum Generation in Transparent Solid-State Media (Springer, 2019).

42. M. Kolesik, G. Katona, J. V. Moloney, and E. M. Wright, "Theory and simulation of supercontinuum generation in transparent bulk media," Appl. Phys. B: Lasers Opt. 77, 185-195 (2003).

43. M. Kolesik, G. Katona, J. V. Moloney, and E. M. Wright, "Physical Factors Limiting the Spectral Extent and Band Gap Dependence of Supercontinuum Generation," Phys. Rev. Lett. 91, 043905 (2003).

44. B. Kibler and P. Béjot, "Discretized conical waves in multimode optical fibers," arXiv:2007.04707 [physics.optics] (2020).

45. L. G. Wright, S. Wabnitz, D. N. Christodoulides, and F. W. Wise, "Ultrabroadband dispersive radiation by spatiotemporal oscillation of multimode waves," Phys. Rev. Lett. 115, 223902 (2015).

46. M. Kolesik, E. M. Wright, and J. V. Moloney, "Dynamic nonlinear X waves for femtosecond pulse propagation in water," Phys. Rev. Lett. 92, 253901 (2004).

47. A. Couairon, E. Gaizauskas, D. Faccio, A. Dubietis, and P. D. Trapani, "Nonlinear X-wave formation by femtosecond filamentation in Kerr media," Phys. Rev. E 73, 016608 (2006). 
48. M. A. Porras, A. Dubietis, E. Kučinskas, F. Bragheri, V. Degiorgio, A. Couairon, D. Faccio, and P. Di Trapani, "From X- to O-shaped spatiotemporal spectra of light filaments in water," Opt. Lett. 30, 3398 (2005).

49. D. Faccio, A. Matijosius, A. Dubietis, R. Piskarskas, A. Varanavičius, E. Gaizauskas, A. Piskarskas, A. Couairon, and P. Di Trapani, "Nearand far-field evolution of laser pulse filaments in Kerr media," Phys. Rev. E 72, 037601 (2005). 